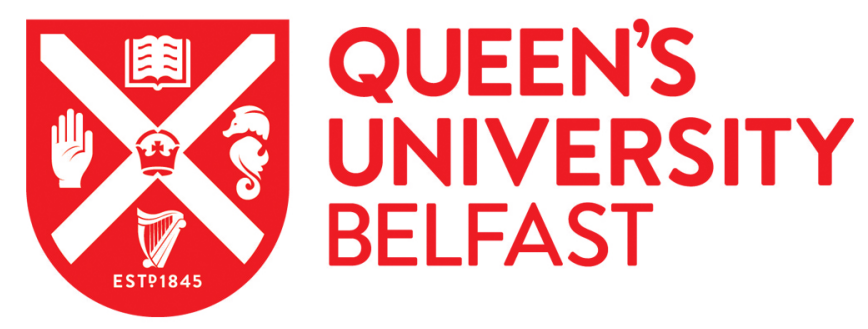

\title{
What Musculoskeletal (MSK) Conditions are Referred from Routine General Practice (GP) and what Impact does this have on Developing Innovative Care Models for Patients with MSK Conditions in Primary
}

\section{Care?}

Heron, N., \& Ryans, I. (2016). What Musculoskeletal (MSK) Conditions are Referred from Routine General Practice (GP) and what Impact does this have on Developing Innovative Care Models for Patients with MSK Conditions in Primary Care? International Journal of Physical Medicine and Rehabilitation, 4(375), [100375]. https://doi.org/10.4172/2329-9096.1000375

Published in:

International Journal of Physical Medicine and Rehabilitation

Document Version:

Publisher's PDF, also known as Version of record

Queen's University Belfast - Research Portal:

Link to publication record in Queen's University Belfast Research Portal

\section{Publisher rights}

(C) 2016 Heron N, et al. This is an open access article published under a Creative Commons Attribution License

(https://creativecommons.org/licenses/by/4.0/), which permits unrestricted use, distribution and reproduction in any medium, provided the author and source are cited.

\section{General rights}

Copyright for the publications made accessible via the Queen's University Belfast Research Portal is retained by the author(s) and / or other copyright owners and it is a condition of accessing these publications that users recognise and abide by the legal requirements associated with these rights.

Take down policy

The Research Portal is Queen's institutional repository that provides access to Queen's research output. Every effort has been made to ensure that content in the Research Portal does not infringe any person's rights, or applicable UK laws. If you discover content in the Research Portal that you believe breaches copyright or violates any law, please contact openaccess@qub.ac.uk. 


\title{
International Journal of Physical Medicine \& Rehabilitation
}

\section{What Musculoskeletal (MSK) Conditions are Referred from Routine General Practice (GP) and what Impact does this have on Developing Innovative Care Models for Patients with MSK Conditions in Primary Care?}

\section{Neil Heron ${ }^{1,2^{*}}$ and lan Ryans ${ }^{1}$}

${ }^{1}$ Centre for Public Health, School of Medicine, Dentistry and Biomedical Science, Institute of Clinical Science B, Royal Victoria Hospital, Queen's University Belfast, Room 01012, Grosvenor Road, Belfast BT12 6BA, UK

${ }^{2}$ UKCRC Centre of Excellence for Public Health (Northern Ireland), Institute of Clinical Science B, Royal Victoria Hospital, Grosvenor Road, Belfast BT12 6BA, UK "Corresponding author: Neil Heron, Level 4-Department of General Practice, Dunluce Health Centre, 1 Dunluce Avenue, Belfast, BT9 7HR, UK, Tel: 07917716425; Email: nheron02@qub.ac.uk

Received date: October 17, 2016; Accepted date: October 26, 2016; Published date: November 01, 2016

Copyright: (C) 2016 Heron N, et al. This is an open-access article distributed under the terms of the Creative Commons Attribution License, which permits unrestricted use, distribution, and reproduction in any medium, provided the original author and source are credited.

\begin{abstract}
Introduction: The current ethos within the United Kingdom (UK) health system is to encourage community management of health problems, increasing primary care workload. Yet General Practice (GP) is currently in 'crisis' with significant workload pressures. GP Federations have been developed to allow more collaborative working between GP practices and help develop new innovative models of care to better manage the GP workload pressures.
\end{abstract}

Musculoskeletal(MSK) conditions constitute approximately $20 \%$ of General Practice(GP) consultations and therefore the Belfast GP Federation aimed to assess the demand for MSK conditions to allow development of new primary care-based treatment pathways for these conditions. The aim of this paper is therefore to assess the demand for orthopaedic, rheumatology and chronic MSK painful conditions by assessing the referrals from 2 GP practices and the referrals to one orthopaedic Integrated Clinical Assessment and Treatment services (ICATs) clinic for these conditions and then propose innovative models of care to manage this demand within the community.

Methods: Secondary care referral rates for two urban GP surgeries in the Belfast area were assessed in April, 2016 to orthopaedics, rheumatology and chronic pain clinics. The referrals to an orthopaedic ICATs clinics, staffed by one GPwSI in MSK, in May 2016 were also reviewed. The orthopaedic ICATs team receive referrals from GPs regarding musculoskeletal conditions and this particular service is based in the Southern Trust area of Northern Ireland.

Results: Overall from the 2 GP surgeries there was 59 orthopaedic referrals, 11 to rheumatology and 3 to the chronic pain clinic. The commonest joint referred to the orthopaedic clinic was knee (15 referrals, $25.4 \%$ ) and the commonest reason to refer to rheumatology was to exclude an inflammatory arthritis ( 6 referrals, $54.5 \%)$. There was then 25 referrals to ICATs, with the commonest reason for referral being neck (6 referrals, $24 \%$ ) and back (4 referrals, $16 \%)$. The commonest treatment options employed within the ICAT service included joint injections (8 patients, $32 \%$ ) with referral to in-house physiotherapy (8 patients, $32 \%$ ).

Conclusions: UK GP is currently under significant workload pressures and musculoskeletal conditions, including orthopaedic, rheumatology and chronic painful conditions, make up a significant proportion of this workload. The main musculoskeletal areas which GPs are referring to secondary care include knee and spinal conditions. To help better manage this workload within primary care we propose developing a new community-based monthly musculoskeletal clinic based within local GP surgeries, supported by a MSK educational programme open to all GPs in the area. Outcomes which will be monitored from this quality improvement work will include secondary care referrals and maintaining high patient satisfaction as well as improving GP confidence in managing MSK conditions.

Keywords: Musculoskeletal; Primary care; Innovative care pathways; Reducing referrals

\section{What We Already Know}

Musculoskeletal (MSK) conditions make up approximately $20 \%$ of the GP workload.

GPs often do not feel comfortable in managing MSK conditions and therefore a high referral burden to secondary care often results.
Within the UK health system, there is now an emphasis on shifting patient management from secondary and tertiary care into the community, with subsequent management pressures for community health workers, including GPs. New service models are needed to manage this demand.

\section{What This Study Adds}

The main MSK areas which GPs are referring to secondary care include knee and spinal conditions. 
Citation: Heron N, Ryans I (2016) What Musculoskeletal (MSK) Conditions are Referred from Routine General Practice (GP) and what Impact does this have on Developing Innovative Care Models for Patients with MSK Conditions in Primary Care?. Int J Phys Med Rehabil 4: 375. doi:10.4172/2329-9096.1000375

Page 2 of 10

The commonest community-based treatment options employed for MSK conditions are corticosteroid/local anaesthetic joint injections with advise regarding exercise prescription, which may or may not be with a physiotherapist.

To help better manage the MSK workload within primary care we propose developing a new community-based monthly MSK clinic based within local GP surgeries, supported by a MSK educational programme open to all GPs in the area.

\section{Introduction}

Musculoskeletal (MSK) conditions make up a significant workload within general practice (GP), with one in seven GP consultations reported to be for MSK conditions [1]. The current ethos within the UK healthcare system is for a 'shift left' in patient management and within Northern Ireland there is the Transforming Your Care policy [2], with more and more medical conditions being managed within the community. Yet GP within the UK and in Northern Ireland is in 'crisis' $[3,4]$, with significant workload pressures on those who work within the community and in the primary care team. The onus is therefore on those working within GP and the community to develop innovative models of care, breaking down the traditional barriers between primary and secondary care, allowing more effective management of the workload within primary care by developing integrative models of care. One way to do this is to invest in GP Federations [4,5], a GP Federation being a collection of practices and primary care teams who work together, "developing and delivering high quality, patient-focused services for their local communities" [4] delivering care to approximately 20 GP practices and 100,000 patients [5]. Such primary care investment and collective working is in keeping with the recent call from the Northern Ireland General Practitioners Committee of the British Medical Association (BMA) to better support NI GP and avert this 'crisis' [3].

With this in mind, the Belfast GP Federation has appointed 2 MSK clinical leads to develop innovative models of care for patients with orthopaedic, rheumatology and chronic MSK painful conditions. The first part of this role is therefore to assess the demand for these conditions within the community to allow the Federation to better plan the innovative models of care for musculoskeletal patients. The aim of this paper is therefore to assess the demand for orthopaedic, rheumatology and chronic MSK painful conditions by assessing the referrals from 2 GP practices and the referrals to one orthopaedic Integrated Clinical Assessment and Treatment services (ICATs) clinic for these conditions and then propose innovative models of care to manage this demand within the community.

\section{Methods}

Secondary care MSK referral rates for two GP surgeries in the Belfast area were assessed in April, 2016 to orthopaedics, rheumatology and chronic pain clinics. Practice 1 serves a patient population of approximately 9,000 and has 5 GP partners and 1 salaried GP. Practice 2 serves a patient population of approximately 6,900 and has 3 GP partners and 1 salaried GP. Both practices use Egton Medical Information Systems (EMIS) computer systems, are urban GP surgeries and have one partner with a specialist interest in the area of primary care musculoskeletal conditions. The referrals to 5 orthopaedic ICATs clinics, staffed by one GPwSI in MSK, in May 2016 were also reviewed. The orthopaedic ICATs team receive referrals from GPs about patients with musculoskeletal conditions, being community-based clinics (straddling the divide between primary and secondary care) and this particular service serves the Southern Trust area of Northern Ireland. Within the ICATs service, the doctor can manage the patients themselves (e.g. with joint injections, exercise advice) or refer to colleagues within the service including extendedscope physiotherapists and podiatry. If the condition cannot be managed within the ICATs service then a secondary care referral will occur. For physiotherapy services, the patient can be referred to ICATs physiotherapy, which offers specialist musculoskeletal physiotherapy, or to 'core' physiotherapy which provides general physiotherapy services. No ethical approval was required for this study as it an audit of musculoskeletal conditions referred to secondary care.

\section{Results}

\section{Practice 1 referrals}

On reviewing referrals to orthopaedics, rheumatology and the pain clinics from this surgery in April 2016, there were 37, 4 and 0 referrals, respectively. The orthopaedic referrals (Table 1) included 9 for knee osteoarthritis, 6 with back pain, 5 paediatric cases (2 abnormal gaits with 1 each of clicking hip, suspected brachial plexus injury at 8 week check, and fixed flexion deformity of finger at 8 week assessment), 4 with neck pain, 3 hip osteoarthritis, 3 shoulder issues (confirmed bilateral rotator cuff tear, suspected bicipital tendonitis, and glenohumeral osteoarthritis), 2 acute knee injuries, and one each of trigger finger, trochanteric bursa, carpal tunnel syndrome, osteoporosis assessment and plantar fasciitis. The 4 rheumatology referrals (Table 2) included 2 to exclude an inflammatory arthritis and one each for carpal tunnel syndrome and steroid/local anaesthetic injections for widespread hand osteoarthritis.

\begin{tabular}{|c|c|c|c|c|c|}
\hline Patient & Reason for referral & Notes & Ix to date & Treatment to date & Alternative options to referral \\
\hline 1 & $\begin{array}{lr}\text { Left } & \text { knee } \\
\text { osteoarthritis } & (\mathrm{OA})- \\
\text { query need } & \text { for } \\
\text { replacement } & \end{array}$ & $\begin{array}{l}\text { Had been seen orthopaedic } \\
\text { consultant on } 2 \text { occasions+course of } \\
\text { physiotherapy }\end{array}$ & $\begin{array}{l}\text { Knee X-ray-severe } \\
\text { OA }\end{array}$ & $\begin{array}{l}\text { Oral (PO) analgesia, ortho } \\
\text { r/w, physio input. }\end{array}$ & $\begin{array}{l}\text { Intra-articular (IA) steroid/local } \\
\text { anesthesia (LA) injections. }\end{array}$ \\
\hline 2 & Bilateral hip OA & - & Hip X-ray & $\begin{array}{l}\mathrm{PO} \text { analgesia, IA steroid/LA } \\
\text { injections, physio }\end{array}$ & Nil \\
\hline 3 & $\begin{array}{l}\text { Bilateral knee } \\
\text { query need } \\
\text { replacement }\end{array}$ & - & $\begin{array}{l}\text { Knee X-ray - } \\
\text { severe OA }\end{array}$ & $\begin{array}{l}\text { PO analgesia, physio, } \\
\text { steroid/LA injections }\end{array}$ & Nil \\
\hline
\end{tabular}


Citation: Heron N, Ryans I (2016) What Musculoskeletal (MSK) Conditions are Referred from Routine General Practice (GP) and what Impact does this have on Developing Innovative Care Models for Patients with MSK Conditions in Primary Care?. Int J Phys Med Rehabil 4: 375. doi:10.4172/2329-9096.1000375

Page 3 of 10

\begin{tabular}{|c|c|c|c|c|c|}
\hline 4 & $\begin{array}{lr}\text { Right } & \text { knee } \\
\text { osteoarthritis } & (\mathrm{OA})- \\
\text { query need } & \text { for } \\
\text { replacement } & \end{array}$ & - & $\begin{array}{l}\text { Knee X-ray-OA } \\
\text { changes }\end{array}$ & $\begin{array}{l}\text { PO analgesia, physio, IA } \\
\text { steroid/LA injections }\end{array}$ & Nil \\
\hline 5 & Sciatica & Physio requesting referral to ortho & - & PO analgesia, physio & Nil \\
\hline 6 & $\begin{array}{l}\mathrm{L} \text { 3rd trigger finger- } \\
\text { query need for } \\
\text { steroid/LA injection }\end{array}$ & - & - & Nil & Injection in practice \\
\hline 7 & $\begin{array}{l}\text { Right carpal tunnel } \\
\text { syndrome symptoms }\end{array}$ & $\begin{array}{l}\text { GP querying need for steroid } \\
\text { injection further investigation }\end{array}$ & - & Nil & Steroid injection in practice \\
\hline 8 & Bilateral knee pains & $\begin{array}{l}\text { Knee xray report-mild OA changes; ? } \\
\text { osteochondritis dissecans defect in } \\
\text { the medial femoral condyle with } \\
\text { query loose body-advised referral for } \\
\text { MRI }\end{array}$ & - & Po analgesia & $\begin{array}{l}\text { Option-get MRI 1st-if confirmed } \\
\text { xray findings, then refer on }\end{array}$ \\
\hline 9 & $\begin{array}{l}\text { Osteoporosis } \\
\text { assessment }\end{array}$ & On PO steroids with diagnosis of RA & - & - & $\begin{array}{l}\text { Refer for DEXA scan; blood } \\
\text { profile in primary care; start } \\
\text { vitamin } \\
\text { supplementation; D/calcium } \\
\text { bisphosphonate if appropriate }\end{array}$ \\
\hline 10 & $\begin{array}{l}\text { Spinal stenosis on } \\
\text { MRI of lumbar spine }\end{array}$ & - & $\begin{array}{l}\text { MRI-spinal } \\
\text { stenosis at } \mathrm{L} 4-5, \\
\text { X-ray-OA } \\
\text { changes, blood } \\
\text { screen }\end{array}$ & PO analgesia, physio & Nil \\
\hline 11 & $\begin{array}{lr}\text { Right } & \text { knee } \\
\text { osteoarthritis } & (\mathrm{OA})- \\
\text { query need } & \text { for } \\
\text { replacement } & \end{array}$ & Also referred to falls clinic & $\begin{array}{l}\text { Knee X-ray-OA } \\
\text { changes }\end{array}$ & PO analgesia. & $\begin{array}{l}\text { Physio referral, steroid/LA } \\
\text { injection }\end{array}$ \\
\hline 12 & Abnormal gait & 5 ууо & $\begin{array}{l}\text { Referred through } \\
\text { GP but had } \\
\text { already attended } \\
\text { A\&E }\end{array}$ & Nil & $\begin{array}{l}\text { Referral rejected }-? \text { transient } \\
\text { synovitis-refer if not settling/any } \\
\text { concerns }\end{array}$ \\
\hline 13 & $\begin{array}{l}\text { Right arm } \\
\text { abnormality in an } 8 \\
\text { week old }\end{array}$ & Query brachial plexus nerve injury & $\begin{array}{l}\text { Picked up at } 8 \\
\text { week assessment } \\
\text { by GP }\end{array}$ & Nil & Nil \\
\hline 14 & Left knee pain & - & $\begin{array}{l}\text { Referred for X-ray- } \\
\text { no result available }\end{array}$ & $\begin{array}{l}\text { PO analgesia, IA steroid/LA } \\
\text { injection }\end{array}$ & $\begin{array}{l}\text { Refer for IA steroid/LA injection } \\
\text { and then course of physio first } \\
\text { before referring to orthopaedics }\end{array}$ \\
\hline 15 & $\begin{array}{l}\text { Left knee injury } 10 \\
\text { weeks ago-clinically } \\
\text { left medial collateral } \\
\text { ligament medial } \\
\text { meniscus injury }\end{array}$ & No symptoms of locking or instability & Referred for X-ray & - & $\begin{array}{l}\text { Xray and then acute } \\
\text { management (POLICE) with } \\
\text { follow-up before referring for } \\
\text { physio }\end{array}$ \\
\hline 16 & $\begin{array}{l}\text { Right anterior } \\
\text { shoulder/bicep pain }\end{array}$ & $\begin{array}{l}\text { Shoulder ultrasound, shoulder and } \\
\text { elbow X-ray normal }\end{array}$ & $\begin{array}{l}\text { Referred-query } \\
\text { cause of pain }\end{array}$ & $\begin{array}{l}\text { PO analgesia, exercise } \\
\text { advice }\end{array}$ & Nil \\
\hline 17 & $\begin{array}{l}\text { Neck pain - referred } \\
\text { for query any } \\
\text { injection therapies } \\
\text { available }\end{array}$ & Neck X-ray-OA changes & - & $\begin{array}{l}\text { PO analgesia; titrating up } \\
\text { analgesic ladder }\end{array}$ & Refer to physio first \\
\hline 18 & $\begin{array}{l}\text { Acute neck pain after } \\
\text { game of golf }\end{array}$ & - & $\begin{array}{l}\text { Blood screen } \\
\text { undertaken in GP }\end{array}$ & $\begin{array}{l}\text { PO analgesia; already seen } \\
\text { chiropractornad }\end{array}$ & $\begin{array}{l}\text { Refer to physio first; titrate } \\
\text { analgesia }\end{array}$ \\
\hline 19 & $\begin{array}{l}\text { Chronic neck pain- } \\
\text { years }\end{array}$ & X-ray and MRI-mild oa changes & - & PO analgesia; physio & Nil \\
\hline 20 & $\begin{array}{l}\text { Neck pain with } \\
\text { radiculopathy } \\
\text { symptoms }\end{array}$ & $\begin{array}{l}\text { X-ray showed advanced oa changes- } \\
\text { referred for MRI and ortho r/w }\end{array}$ & - & $\mathrm{PO}$ analgesia & $\begin{array}{l}\text { Refer to physio first with MRI } \\
\text { referral }\end{array}$ \\
\hline
\end{tabular}


Citation: Heron N, Ryans I (2016) What Musculoskeletal (MSK) Conditions are Referred from Routine General Practice (GP) and what Impact does this have on Developing Innovative Care Models for Patients with MSK Conditions in Primary Care?. Int J Phys Med Rehabil 4: 375. doi:10.4172/2329-9096.1000375

Page 4 of 10

\begin{tabular}{|c|c|c|c|c|c|}
\hline 21 & $\begin{array}{l}\text { Right hip OA-query } \\
\text { need for hip } \\
\text { replacement }\end{array}$ & $\begin{array}{l}\text { Right hip X-ray-advanced OA } \\
\text { changes }\end{array}$ & - & PO analgesia, physio & Nil \\
\hline 22 & $\begin{array}{l}\text { Right trochanteric } \\
\text { bursa }\end{array}$ & Referring for steroid/LA injection & - & PO analgesia & $\begin{array}{l}\text { Steroid injection in practice } \\
\text { physio referral }\end{array}$ \\
\hline 23 & $\begin{array}{l}\text { Left shoulder OA on } \\
\text { X-ray-minor }\end{array}$ & - & - & - & $\begin{array}{l}\text { Analgesia, steroid/LA injection, } \\
\text { physio }\end{array}$ \\
\hline 24 & Bilateral hip OA & $\begin{array}{l}\text { Referring for query steroid/LA } \\
\text { injections }\end{array}$ & - & $\begin{array}{l}\text { Bilateral hip X-ray-OA } \\
\text { changes }\end{array}$ & $\begin{array}{l}\text { Steroid/LA injections in practice } \\
\text { with physio therapy }\end{array}$ \\
\hline 25 & $\begin{array}{l}\text { Bilateral knee } \\
\text { query need } \\
\text { replacement }\end{array}$ & $\begin{array}{l}\text { Bilateral knee X-ray-severe OA } \\
\text { changes }\end{array}$ & - & $\begin{array}{l}\text { Knee IA steroid/LA injections, } \\
\text { physio }\end{array}$ & Nil \\
\hline 26 & $\begin{array}{lr}\text { Right } & \text { knee } \\
\text { osteoarthritis } & (\mathrm{OA})- \\
\text { query need } & \text { for } \\
\text { replacement } & \end{array}$ & X-ray-moderate OA changes & - & - & $\begin{array}{l}\text { Knee steroid/LA injection in } \\
\text { practice, physio }\end{array}$ \\
\hline 27 & $\begin{array}{l}\text { MRI-showed spinal } \\
\text { stenosis, no focal } \\
\text { neurology }\end{array}$ & - & - & - & $\begin{array}{l}\text { PO analgesia and physio } \\
\text { referral first with advice about } \\
\text { red flags and when to seek } \\
\text { urgent review }\end{array}$ \\
\hline 28 & $\begin{array}{l}2 \mathrm{yr} h \mathrm{hx} \text { of sciatica } \\
\text { with focal neurology } \\
\text { reported }\end{array}$ & $\begin{array}{l}\text { Previously had physio-no } \\
\text { improvement }\end{array}$ & - & $\begin{array}{l}\text { Lumbar spine } \quad \text { xray-OA } \\
\text { changes }\end{array}$ & Nil \\
\hline 29 & $\begin{array}{lr}\text { Right } & \text { knee } \\
\text { osteoarthritis } & (\mathrm{OA})- \\
\text { query need } & \text { for } \\
\text { replacement } & \end{array}$ & $\begin{array}{l}\text { Previously had IA steroid/LA } \\
\text { injections }\end{array}$ & $\begin{array}{l}\text { Now on strong } \\
\text { opiate meds. } \\
\text { Declined physio }\end{array}$ & $\begin{array}{l}\text { Right knee xray-severe OA } \\
\text { changes }\end{array}$ & Nil \\
\hline 30 & $\begin{array}{l}\text { Back pain-had been } \\
\text { told by orthopaedic } \\
\text { consultant to be } \\
\text { referred for steroid } \\
\text { injection }\end{array}$ & - & - & - & $\begin{array}{l}\text { Physio referral first with then } \\
\text { onward referral if not settling }\end{array}$ \\
\hline 31 & $\begin{array}{l}\text { Twisting injury to left } \\
\text { knee } 8 \text { weeks ago-? } \\
\text { meniscal tear }\end{array}$ & Referred for X-ray & $\begin{array}{l}\text { No locking or } \\
\text { instability } \\
\text { symptoms }\end{array}$ & - & $\begin{array}{l}\text { Physio, PO analgesia- refer to } \\
\text { orthopaedics if not settling }\end{array}$ \\
\hline 32 & $\begin{array}{l}4 \text { year old with } 6 \\
\text { month history of } \\
\text { clicking hip-maternal } \\
\text { concerns }\end{array}$ & Hip X-ray-NAD & - & - & Nil \\
\hline 33 & $\begin{array}{l}\text { Referred as severe } \\
\text { OA changes on xray } \\
\text { of lumbar spine and } \\
\text { sciatica symptoms- } \\
\text { query need for MRI }\end{array}$ & X-ray of lumbar spine & - & - & $\begin{array}{l}\text { Physio with or without MRI } \\
\text { referral first }\end{array}$ \\
\hline 34 & $\begin{array}{l}\text { Bilateral rotator cuff } \\
\text { tears -query need for } \\
\text { operative repair }\end{array}$ & X-ray, U/S, analgesia, physio & $\begin{array}{l}\text { Subacromial } \\
\text { steroid injections }\end{array}$ & - & Nil \\
\hline 35 & $\begin{array}{l}\text { Left-sided plantar } \\
\text { fasciitis symptoms }\end{array}$ & - & - & - & $\begin{array}{l}\text { Steroid injections in practice } \\
\text { and advise re stretches, etc }\end{array}$ \\
\hline 36 & $\begin{array}{l}\text { Change in gait , } \\
\text { asymmetrical, in } 1 \text { yo }\end{array}$ & - & - & - & $\begin{array}{l}\text { Hip/pelvis xray pre-referral but } \\
\text { would still need referral }\end{array}$ \\
\hline 37 & $\begin{array}{l}\text { Fixed flexion } \\
\text { deformity of finger at } \\
8 \text { wk assessment }\end{array}$ & - & - & - & Nil \\
\hline
\end{tabular}

Table 1: Orthopaedic referrals from Practice 1. 
Citation: Heron N, Ryans I (2016) What Musculoskeletal (MSK) Conditions are Referred from Routine General Practice (GP) and what Impact does this have on Developing Innovative Care Models for Patients with MSK Conditions in Primary Care?. Int J Phys Med Rehabil 4: 375. doi:10.4172/2329-9096.1000375

Page 5 of 10

\begin{tabular}{|c|c|c|c|c|c|}
\hline Patient & Reason for referral & Notes & Ix to date & Treatment to date & Alternative options to referral \\
\hline 1 & $\begin{array}{l}\text { For carpal tunnel steroid } \\
\text { injection }\end{array}$ & & Nil recent & $\begin{array}{l}\text { Carpal tunnel splint provided } \\
\text { following recent A\&E } \\
\text { attendance, PO analgesia }\end{array}$ & $\begin{array}{l}\text { Steroid injection in practice; consider } \\
\text { inflammatory blood screen to exclude } \\
\text { secondary causes }\end{array}$ \\
\hline 2 & $\begin{array}{l}\text { Query inflammatory } \\
\text { arthritis }\end{array}$ & $\begin{array}{l}\text { History of intermittent } \\
\text { swollen joints }+ \text { raised anti- } \\
\text { chromatin level of } 5.6\end{array}$ & $\begin{array}{l}\text { Inflammatory blood } \\
\text { screen }\end{array}$ & PO analgesia & $\begin{array}{l}\text { Also consider x-ray of appropriate areas } \\
\text { with referral }\end{array}$ \\
\hline 3 & $\begin{array}{l}\text { Query need for IA hand } \\
\text { steroid/LA injections }\end{array}$ & Had previously-worked well & $\begin{array}{l}\text { Known psoriatic } \\
\text { arthropathy }\end{array}$ & $\mathrm{PO}$ analgesia & Steroid/LA IA injection in practice \\
\hline 4 & $\begin{array}{l}\text { Query erosive arthritis } \\
\text { changes seen on hand } \\
\text { X-ray }\end{array}$ & $\begin{array}{l}\text { Referred due to X-ray } \\
\text { findings-query inflammatory } \\
\text { arthritis }\end{array}$ & & & Nil \\
\hline
\end{tabular}

Table 2: Rheumatology referrals from Practice 1.

Alternative management options, which could have been considered in practice prior to the secondary care orthopaedic referral included 10 for local anesthetic/steroid injection with or without exercise advice, 10 for physiotherapy and 1 referral each for an MRI and osteoporosis management. Alternative management options for the rheumatology secondary care referrals were to consider steroid/local anaesthetic injections in the GP surgery for 2 of the referrals.

\section{Practice 2 referrals}

On reviewing referrals to orthopaedics, rheumatology and the pain clinics from this surgery in April 2016, there were 22, 7 and 3 referrals, respectively. The orthopaedic referrals (Table 3) included 6 for knee symptoms and 3 each for shoulder symptoms, foot pathology and paediatric cases (one each for flat feet, poor balance and unilateral tiptoe walking). There were then 2 referrals for carpal tunnel syndrome with one each for a wrist ganglion, back pain, neck pain, osteoporosis assessment, and lateral epicondylitis/tennis elbow. Whereas the rheumatology referrals (Table 4) included 4 to exclude an inflammatory arthritis and then 1 each for neck pain with radiculopathy, suspected fibromyalgia and complete supraspinatus tendon rupture in the shoulder. Finally, the 3 pain clinic referrals (Table 5) included 2 for chronic back pain and 1 for chronic neck pain.

\begin{tabular}{|c|c|c|c|c|c|}
\hline Patient & Reason for referral & Notes & Ix to date & Treatment to date & Alternative options to referral \\
\hline 1 & $\begin{array}{l}\text { Ganglion to dorsum of } \\
\text { right wrist }\end{array}$ & History of fibromyalgia & - & - & Aspirate/inject in practice \\
\hline 2 & Left shoulder OA & $\begin{array}{lcr}\begin{array}{l}\text { Previously } \\
\text { steroid/LA }\end{array} & \text { had } & \text { shoulder } \\
\text { injection } & \text { which }\end{array}$ & $\begin{array}{ll}\text { Shoulder } & \text { X-ray- } \\
\text { glenohumeral } & \\
\text { osteoarthritis } & \end{array}$ & $\mathrm{PO}$ analgesia & $\begin{array}{l}\text { Glenohumeral steroid/LA injection in } \\
\text { practice physio }\end{array}$ \\
\hline 3 & Low back pain & - & MRI-OA changes & PO analgesia & $\begin{array}{l}\text { Physio with advice about red flag } \\
\text { features and when to seek urgent } \\
\text { review }\end{array}$ \\
\hline 4 & $\begin{array}{l}\text { Right knee pain-query } \\
\text { meniscal pathology }\end{array}$ & $\begin{array}{l}\text { History of right knee locking and } \\
\text { giving way }\end{array}$ & Right knee X-ray - nad & Po analgesia & - \\
\hline 5 & $\begin{array}{l}\text { Left shoulder } \\
\text { ultrasound-subacromial } \\
\text { bursitis }\end{array}$ & - & Left shoulder ultrasound & Po analgesia & $\begin{array}{l}\text { Subacromial steroid/LA injection in } \\
\text { practice }+/ \text { - physio }\end{array}$ \\
\hline 6 & $\begin{array}{l}\text { Right carpal tunnel } \\
\text { syndrome }\end{array}$ & History of fibromyalgia & - & $\begin{array}{l}\text { Given trial of } \\
\text { amitriptyline }\end{array}$ & Steroid injection in practice hand splints \\
\hline 7 & $\begin{array}{l}\text { Right knee pain-recent } \\
\text { MRI }\end{array}$ & $\begin{array}{l}\text { No history of injury, locking or } \\
\text { instability }\end{array}$ & $\begin{array}{l}\text { MRI-meniscal tear, } \\
\text { osteochondral defect, OA } \\
\text { changes }\end{array}$ & $\mathrm{PO}$ analgesia & Consider physio referral first \\
\hline 8 & $\begin{array}{l}6 \text { year old referred for } \\
\text { flat feet and issues with } \\
\text { walking }\end{array}$ & $\begin{array}{l}\text { Seen by podiatrist who had } \\
\text { advised referral }\end{array}$ & - & - & $\begin{array}{l}\text { Referral returned by orthopaedics - } \\
\text { inappropriate referral-advised normal } \\
\text { variant }\end{array}$ \\
\hline 9 & $\begin{array}{l}1 \text { year old with history } \\
\text { of poor balance and } \\
\text { falls-parental concern }\end{array}$ & - & - & - & $\begin{array}{l}\text { Referral returned by orthopaedics - } \\
\text { inappropriate referral-advised normal } \\
\text { variant }\end{array}$ \\
\hline
\end{tabular}


Citation: Heron N, Ryans I (2016) What Musculoskeletal (MSK) Conditions are Referred from Routine General Practice (GP) and what Impact does this have on Developing Innovative Care Models for Patients with MSK Conditions in Primary Care?. Int J Phys Med Rehabil 4: 375. doi:10.4172/2329-9096.1000375

Page 6 of 10

\begin{tabular}{|c|c|c|c|c|c|}
\hline 10 & $\begin{array}{l}\text { Neck pain with } \\
\text { radiculopathy } \\
\text { symptoms }\end{array}$ & $\begin{array}{l}\text { MRI-showed cervical disc } \\
\text { prolapse }\end{array}$ & MRI & $\mathrm{PO}$ analgesia & $\begin{array}{l}\text { Query physio first with pain } \\
\text { management in practice }\end{array}$ \\
\hline 11 & $\begin{array}{l}\text { History of left knee } \\
\text { locking for } 2 \text { months- } \\
\text { patient can force the } \\
\text { knee to straighten }\end{array}$ & X-ray-normal & - & $\mathrm{PO}$ analgesia & $\begin{array}{l}\text { MRI, physio, with advice if acute locking } \\
\text {-A\&E }\end{array}$ \\
\hline 12 & $\begin{array}{l}\text { Referred for } \\
\text { osteoporosis } \\
\text { assessment }\end{array}$ & $\begin{array}{l}\text { Had previous DEXA scan which } \\
\text { advised repeat in } 3 \text { years }\end{array}$ & Bone scan-osteopenia & $\begin{array}{l}\text { No } t / \text { ment to date- } \\
\text { advised lifestyle } \\
\text { measures. }\end{array}$ & $\begin{array}{l}\text { Refer for DEXA scan; blood profile in } \\
\text { primary care; start vitamin D/calcium } \\
\text { supplementation; start bisphosphonate } \\
\text { if appropriate }\end{array}$ \\
\hline 13 & Left knee OA & $\begin{array}{l}\text { Left knee X-ray-mild } \mathrm{OA} \\
\text { changes }\end{array}$ & X-ray & $\mathrm{PO}$ analgesia & $\begin{array}{l}\text { Physio; activity modification; steroid/LA } \\
\text { injection for pain }\end{array}$ \\
\hline 14 & $\begin{array}{l}\text { Right knee pain for } 3 \\
\text { years following a } \\
\text { twisting injury-query } \\
\text { meniscal injury }\end{array}$ & $\begin{array}{l}\text { No history of locking or } \\
\text { instability }\end{array}$ & X-ray & PO analgesia & $\begin{array}{l}\text { Physio 1st IA steroid/LA injection for } \\
\text { pain; if not settling-orthopaedic referral }\end{array}$ \\
\hline 15 & $\begin{array}{ll}\text { Right } & \text { lateral } \\
\text { epicondylitis } & \end{array}$ & - & - & PO analgesia & $\begin{array}{l}\text { Physio novel injection therapies, e.g. } \\
\text { whole blood }\end{array}$ \\
\hline 16 & $\begin{array}{l}\text { Left shoulder pain-AC } \\
\text { joint OA calcification } \\
\text { within rotator cuff } \\
\text { muscles }\end{array}$ & - & Left shoulder X-ray & $\begin{array}{l}\text { Subacromial } \\
\text { steroid injection; } \\
\text { course of physio }\end{array}$ & $\begin{array}{l}\text { For further subacromial steroid/LA } \\
\text { injection and then refer if not settling }\end{array}$ \\
\hline 17 & $\begin{array}{l}\text { Unilateral tip-toe } \\
\text { walking in } 1 \text { year old }\end{array}$ & - & - & - & - \\
\hline 18 & Bilateral hallux valgus & - & X-ray & - & Podiatry referral first \\
\hline 19 & $\begin{array}{l}\text { Right carpal tunnel } \\
\text { syndrome }\end{array}$ & - & $\begin{array}{l}\text { Previously seen by } \\
\text { rheumatology } \\
\text { consultant-2 injections- } \\
\text { advised if symptoms } \\
\text { recur to have surgery }\end{array}$ & - & - \\
\hline 20 & Foot pain, bilateral & $\begin{array}{l}\text { History of bilateral talipes } \\
\text { equinous varus, surgically } \\
\text { corrected; previously had R } \\
\text { calcaneal cuboid fusion }\end{array}$ & $\begin{array}{l}\text { Previously seen by foot } \\
\text { surgeon }\end{array}$ & X-ray & $\begin{array}{l}\text { Query trial of podiatry first before } \\
\text { referral back to foot surgeon }\end{array}$ \\
\hline 21 & $\begin{array}{l}\text { Left knee pain-no } \\
\text { trauma }\end{array}$ & Left knee X-ray-nil; 53 years old & X-ray & $\mathrm{PO}$ analgesia & Physio IA steroid/LA injection \\
\hline 22 & $\begin{array}{l}\text { Right bunion (hallux } \\
\text { valgus) }\end{array}$ & 28 years old & Right foot X-ray & Nil & Podiatry first \\
\hline
\end{tabular}

Table 3: Orthopaedic referrals from Practice 2.

\begin{tabular}{|l|l|l|l|l|}
\hline Patient & Reason for referral & Notes & Ix to date & Treatment to date \\
\hline 1 & Query inflammatroy arthritis & - & $\begin{array}{l}\text { Inflammatory blood screen; X- } \\
\text { ray-DIP 2nd finger swelling }\end{array}$ & PO analgesia \\
\hline 2 & Query inflammatory arthritis & $\begin{array}{l}\text { History } \\
\text { Raynaud's; } \begin{array}{r}\text { of } \\
\text { anti- }\end{array}\end{array}$ & - & Po analgesia \\
\hline 3 & $\begin{array}{l}\text { Neck pain-MRI shows prolapsed } \\
\text { discs }\end{array}$ & - & MRI & PO analgesia \\
\hline 4 & $\begin{array}{l}\text { Widespread pain-suspected } \\
\text { firbomylagia }\end{array}$ & - & $\begin{array}{l}\text { Inflammatory blood screen- } \\
\text { normal }\end{array}$ & Po analgesia \\
\hline
\end{tabular}


Citation: Heron N, Ryans I (2016) What Musculoskeletal (MSK) Conditions are Referred from Routine General Practice (GP) and what Impact does this have on Developing Innovative Care Models for Patients with MSK Conditions in Primary Care?. Int J Phys Med Rehabil 4: 375. doi:10.4172/2329-9096.1000375

Page 7 of 10

\begin{tabular}{|l|l|l|l|l|}
\hline 5 & $\begin{array}{l}\text { Joint erosions seen on X-ray - } \\
\text { query inflammatory arthropathy }\end{array}$ & $\begin{array}{l}\text { X-ray, inflammatory blood } \\
\text { screen }\end{array}$ & PO analgesia \\
\hline 6 & $\begin{array}{l}\text { History of psoriasis and joint } \\
\text { erosions seen on hand X-ray }\end{array}$ & $\begin{array}{l}\text { X-ray, inflammatory blood } \\
\text { screen }\end{array}$ & Po analgesia \\
\hline 7 & Right shoulder pain & - & $\begin{array}{l}\text { Right shoulder X-ray-normal; } \\
\text { right shoulder U/S - complete } \\
\text { rupture of supraspinatus }\end{array}$ & $\begin{array}{l}\text { Ponalgesia, physio, x3 } \\
\text { steroid injections }\end{array}$ \\
\hline
\end{tabular}

Table 4: Rheumatology referrals from Practice 2.

\begin{tabular}{|l|l|l|l|l|}
\hline Patient & Reason for referral & Notes & Ix to date & Treatment to date \\
\hline 1 & $\begin{array}{l}\text { Chronic back pain-recent } \\
\text { decompression surgery }\end{array}$ & - & X-ray, MRI-OA changes & $\begin{array}{l}\text { Orthopaedic input; on gabapentin, } \\
\text { naproxen }\end{array}$ \\
\hline 2 & $\begin{array}{l}\text { Chronic back pain with } \\
\text { padiation management tochniques }\end{array}$ & - & X-ray-OA changes & Started on gabapentin \\
\hline 3 & $\begin{array}{l}\text { Chronic neck pain with } \\
\text { radiculopathy }\end{array}$ & - & X-ray, MRI-OA changes & $\begin{array}{l}\text { Started on co-codamol } \\
\text { diazepam }\end{array}$ \\
\hline
\end{tabular}

Table 5: Chronic Pain Clinic referrals from Practice 2.

Alternative management options which could have been considered in practice prior to the secondary care orthopaedic referrals included 7 for local anaesthetic/steroid injection with or without exercise advice, 5 for physiotherapy, 3 for podiatry and 1 osteoporosis assessment. Whereas the alternative to rheumatology secondary care referrals included 2 to physiotherapy and 1 to the orthopaedic shoulder consultant to consider operative repair of the complete rotator cuff tear. Finally, the chronic pain referrals could have been alternatively managed with in-house analgesic titration with a pain management course/techniques.

\section{Orthopaedic ICAT clinic referrals}

There were 25 referrals (Table 6) to orthopaedic ICATs received in May 2016 to one GPwSI, managed over 5 clinics. The presenting issue included 6 with neck pain and 4 each of back pain, hip pain and carpal tunnel syndrome. There were then 2 referrals each for trigger finger and shoulder symptoms with one each for neck pain, Dupytren's contracture and wrist pain. Treatment within the ICATs service included 8 receiving steroid/local anaesthetic injections to the affected area with or without exercise prescription, 8 referred to the ICATs physio, with 3 referred to core physiotherapy. Two patients were also referred for an MRI of the affected area with one patient each receiving an ultrasound guided steroid/local anaesthetic injection, hand splints and exercise prescription with advice about their condition. Only 4 patients were referred onwards from the ICATs service, with 3 referred to core physiotherapy and 1 for an ultrasound guided steroid/local anesthetic injection.

\begin{tabular}{|l|l|l|l|l|}
\hline Patient & Referral reason & Investigations & Management in ortho ICATs & Onward referral from ICATs \\
\hline 1 & Left knee OA & $\begin{array}{l}\text { Left knee X-ray-OA } \\
\text { changes }\end{array}$ & Steroid/LA IA knee injection; walking stick & No \\
\hline 2 & Back pain/sciatica & Nil & $\begin{array}{l}\text { Core physio referral; trial of neuropathic analgesic } \\
\text { (e.g. amitriptyline) }\end{array}$ & Yes-core physio \\
\hline 3 & $\begin{array}{l}\text { Right hip pain-suspected } \\
\text { labral tear }\end{array}$ & MRI with contrast & $\begin{array}{l}\text { Suspected right labral hip tear trochanteric bursitis } \\
\text { of right hip; referred to ICATs physio }\end{array}$ & No \\
\hline 4 & Neck pain & MRI-normal & $\begin{array}{l}\text { Normal MRI; patient reporting neck pain with } \\
\text { radiation down arm; trial of neuropathic analgesia }\end{array}$ & Yes-core physio \\
\hline 5 & $\begin{array}{l}\text { Thoracic back pain } \\
\text { Right carpal tunnel }\end{array}$ & $\begin{array}{l}\text { MRI-degenerative } \\
\text { changes at T7-T8 }\end{array}$ & $\begin{array}{l}\text { Advised re appropriate strengthening exercises for } \\
\text { back and referred to ICATs physio for input }\end{array}$ & No \\
\hline 7 & $\begin{array}{l}\text { Right middle trigger finger } \\
\text { syndrome }\end{array}$ & $\begin{array}{l}\text { Referred for ultrasound } \\
\text { guided steroid injection } \\
\text { as nodule palpable on the } \\
\text { tendon }\end{array}$ & $\begin{array}{l}\text { Referred for u/s guided steroid injection } \\
\text { Carpal tunnel steroid injection; splints }\end{array}$ & Yes-u/s guided steroid injection \\
\hline
\end{tabular}


Citation: Heron N, Ryans I (2016) What Musculoskeletal (MSK) Conditions are Referred from Routine General Practice (GP) and what Impact does this have on Developing Innovative Care Models for Patients with MSK Conditions in Primary Care?. Int J Phys Med Rehabil 4: 375. doi:10.4172/2329-9096.1000375

Page 8 of 10

\begin{tabular}{|c|c|c|c|c|}
\hline 8 & $\begin{array}{l}\text { Twisting injury to right knee } \\
\text { approx. } 9 \text { months ago }\end{array}$ & $\begin{array}{l}\text { Referred for MRI - ? } \\
\text { meniscus ACL injury }\end{array}$ & MRI referral; ICATs physio & Yes-MRI referral for right knee \\
\hline 9 & $\begin{array}{l}\text { Recurrent left patella } \\
\text { dislocation }\end{array}$ & Left knee X-ray-normal & $\begin{array}{l}\text { Referred for ICATs physio and advised re core } \\
\text { stability }\end{array}$ & No \\
\hline 10 & $\begin{array}{l}\text { Bilateral Dupytren's } \\
\text { contracture of hands, right } \\
\text { greater than left }\end{array}$ & Nil & $\begin{array}{l}\text { Conservative management advised as flexion } \\
\text { contracture mild }(<20 \text { degrees }) \text { and not interfering } \\
\text { with activities of daily living }\end{array}$ & No \\
\hline 11 & $\begin{array}{l}\text { Left knee pain; MRI } \\
\text { showed a medial meniscal } \\
\text { tear and chondromalacia } \\
\text { patellae }\end{array}$ & $\begin{array}{l}\text { No history of locking or } \\
\text { instability }\end{array}$ & $\begin{array}{l}\text { Titration of PO analgesia and referred to ICATs } \\
\text { physio for appropriate quadriceps, hamstring and } \\
\text { gluteal strengthening exercises }\end{array}$ & No \\
\hline 12 & $\begin{array}{l}\text { U/S of left shoulder } \\
\text { showed rotator cuff } \\
\text { tendonitis and subacromial } \\
\text { bursitis }\end{array}$ & - & $\begin{array}{l}\text { Subacromial steroid/LA injection into } L \text { shoulder } \\
\text { and referred to ICATs physio }\end{array}$ & No \\
\hline 13 & $\begin{array}{l}\text { Right carpal tunnel } \\
\text { syndrome and Dupytren's } \\
\text { contracture }\end{array}$ & Nil & $\begin{array}{l}\text { Right carpal tunnel steroid injection; night splints; } \\
\text { conservative management of Dupytren's } \\
\text { contracture }\end{array}$ & No \\
\hline 14 & $\begin{array}{l}\text { Bilateral carpal tunnel } \\
\text { syndrome, left>right }\end{array}$ & Nil & $\begin{array}{l}\text { Trial of night splints; if no improvement - steroid } \\
\text { injection }\end{array}$ & No \\
\hline 15 & $\begin{array}{l}\text { Bilateral hip OA and } \\
\text { bilateral shoulder } \\
\text { impingement, right }>\text { left }\end{array}$ & Hip X-ray-OA changes & $\begin{array}{l}\text { Steroid/LA injection to right hip and then input from } \\
\text { ICATs physio; own GP had already referred for } \\
\text { bilateral U/S guided subacromial steroid/LA } \\
\text { injections }\end{array}$ & No \\
\hline 16 & $\begin{array}{l}82 \text { year old man with } 3 \\
\text { week history of back pain } \\
\text { and past history of prostate } \\
\text { carcinoma }\end{array}$ & $\begin{array}{l}\text { Referred for MRI of } \\
\text { lumbar spine due to } \mathrm{hx} \text { of } \\
\text { prostate ca }\end{array}$ & $\begin{array}{l}\text { Trial of neuropathic agent (amitriptyline); advised } \\
\text { regarding core stability exercises and referred to } \\
\text { ICATs physio for input }\end{array}$ & No \\
\hline 17 & $\begin{array}{l}12 \text { year history of bilateral } \\
\text { anterior patellofemoral } \\
\text { knee pain, right>left }\end{array}$ & $\begin{array}{l}\text { Bilateral xray of knees - } \\
\text { nil }\end{array}$ & $\begin{array}{l}\text { Advised regarding core stability, particularly } \\
\text { quadriceps, hamstring and gluteal strengthening } \\
\text { exercises; then referred to ICATs physio to support } \\
\text { this }\end{array}$ & No \\
\hline 18 & $\begin{array}{l}\text { Left subacromial shoulder } \\
\text { impingement }\end{array}$ & Left shoulder X-ray - nil & $\begin{array}{l}\text { Left subacromial steroid/LA injection; advised re } \\
\text { rotator cuff strengthening exercises and then } \\
\text { referred to ICATs physio for input }\end{array}$ & No \\
\hline 19 & Bilateral hip pain, right $>$ left & $\begin{array}{l}\text { Bilateral hip/pelvis X-ray - } \\
\text { nad }\end{array}$ & $\begin{array}{l}\text { Tight hip flexors and poor lumbar spine mobility- } \\
\text { referred to ICATs physio }\end{array}$ & No \\
\hline 20 & Chronic lumbar back pain & $\begin{array}{l}\text { MRI-degenerative } \\
\text { changes with potential for } \\
\text { nerve root impingement }\end{array}$ & $\begin{array}{l}\text { Trial of neuropathic analgesia; core stability } \\
\text { exercise advice and referred to ICATs physio to } \\
\text { support }\end{array}$ & No \\
\hline 21 & Bilateral hip pain, left>right & X-ray-OA changes & $\begin{array}{l}\text { Exercise prescription and general advice about } \\
\text { osteoarthritis as a condition }\end{array}$ & No \\
\hline 22 & $\begin{array}{l}\text { Left-sided ulna wrist pain. } \\
\text { Had been referred by core } \\
\text { physio after course of } \\
\text { physio and } x 2 \text { steroid/LA } \\
\text { into wrist joint. }\end{array}$ & $\begin{array}{l}\text { X-ray of wrist-oa } \\
\text { changes, particularly at } \\
\text { the radiocarpal joints. }\end{array}$ & - & $\begin{array}{l}\text { Yes - referred for MRI-query triangular } \\
\text { fibrocartilage injury }\end{array}$ \\
\hline 23 & Right 5 th trigger finger & Nil & $\begin{array}{l}\text { Blind injection of steroid/LA around palpable nodule } \\
\text { at MCP area }\end{array}$ & No \\
\hline 24 & $\begin{array}{l}\text { Bilateral carpal tunnel } \\
\text { syndrome, left }>\text { right }\end{array}$ & Nil & Steroid injection to $L$ carpal tunnel with night splints & No \\
\hline 25 & $\begin{array}{l}\text { Left knee pain-OA } \\
\text { changes on X-ray }\end{array}$ & $\begin{array}{l}\text { MRI done to exclude } \\
\text { meniscal injury }\end{array}$ & $\begin{array}{l}\text { Physio for core strengthening exercises IA } \\
\text { steroid/LA injection if not settling }\end{array}$ & No \\
\hline
\end{tabular}

Table 6: Orthopaedic ICAT referrals. 
Citation: Heron N, Ryans I (2016) What Musculoskeletal (MSK) Conditions are Referred from Routine General Practice (GP) and what Impact does this have on Developing Innovative Care Models for Patients with MSK Conditions in Primary Care?. Int J Phys Med Rehabil 4: 375. doi:10.4172/2329-9096.1000375

Page 9 of 10

\section{Discussion}

Musculoskeletal conditions, including orthopaedic, rheumatology and chronic painful conditions, make up a significant workload for routine GP. Through the analysis which has been performed, it can be seen that knee and hip conditions, particularly osteoarthritis affecting these areas, as well as back and neck pain, shoulder, foot and paediatric orthopaedic pathology are common reasons for onward referral from GP to secondary care. Other common reasons for referral include carpal tunnel syndrome, trigger fingers and the need to exclude an inflammatory arthritis. Common treatment options for these musculoskeletal conditions include steroid/local anesthetic injections to the affected area, exercise prescription with or without physiotherapy input, pain management and information sharing about their condition to enable patient empowerment.

\section{Prevalence of MSK conditions in primary care}

The main joint being referred from primary care in this project was knee, with the commonest pathology detected here being osteoarthritis. This finding is in keeping with previous authors [1] who found the knee and back to be the commonest body regions presenting in GP patients with MSK symptoms. Although other authors reported the back and neck regions as the commonest presenting areas for MSK conditions in primary care [6]. This difference may be explained by the fact that our study is looking at the MSK referrals from practice and not simply reporting the MSK conditions presenting to routine GP. The GPs may therefore be filtering out these other musculoskeletal presentations within their own clinics.

\section{Future plans-quality improvement project to manage MSK conditions in primary care}

From this analysis, to help reduce secondary care referrals for musculoskeletal conditions as well as better manage the workload in primary care from musculoskeletal conditions, 2 options have been proposed. The options have included a monthly specialist musculoskeletal clinic based within the local GP surgeries to run alongside an ongoing educational programme to generally up-skill GPs on musculoskeletal conditions. The next step for developing the monthly specialist musculoskeletal clinic within the GP surgery is to pilot this within 10 local surgeries. The clinic would accept referrals for chronic conditions, including shoulder pathology (adhesive capsulitis, shoulder impingement, acromioclavicular (AC) joint symptoms), knee (with symptoms of locking or instability to be referred directly to orthopaedics), hand (carpal tunnel syndrome, trigger finger, 1st carpometacarpal osteoarthritis, de Quervain's tenosynovitis), elbow (lateral and medial epicondylitis) and a chronic pain management programme. Prior to referral, treatment pathways for common musculoskeletal conditions will be available for the GPs to refer to and the referrer should consider an X-ray of the affected joint with or without an appropriate inflammatory blood screen if clinically indicated. This clinic will be staffed by a local GP with a specialist interest in musculoskeletal conditions with access to physiotherapy, pharmacy, podiatry, psychology and musculoskeletal ultrasound imaging. This approach has been previously trialed with significant success in reducing onward secondary care referrals for musculoskeletal conditions and radiology referrals as well as providing high patient satisfaction, although this approach was only piloted in one surgery $[7,8]$. This innovative community based musculoskeletal clinic would then be supported by a monthly education programme on common musculoskeletal conditions seen within primary care and open to any GP within the Belfast GP Federation to attend. Outcomes which will then be monitored from this quality improvement project will include onward secondary care referrals from the practice and patient satisfaction with the new service as well as improving GP confidence in managing musculoskeletal conditions.

\section{Potential limitations}

This is a review of 2 practices' referrals to orthopaedics, rheumatology and chronic pain clinics over one month along with a review of orthopaedic ICAT referrals to one doctor over one month period. The review therefore may be influenced by the practices and the GPwSI which were reviewed as well as the month of the year which the review was performed. In particular, both practices already had a GP with a specialist interest in MSK conditions working in the practice and the referrals for these conditions may therefore be low compared to GP surgeries without access to such a resource. The review of referrals from the practices is dependent on appropriate coding and the data is therefore limited by the statistics provided to us, which may lead to under- or over-estimating of referral rates.

\section{Conclusion}

Primary care and GP within the UK is currently under significant workload pressures and musculoskeletal conditions, including orthopaedic, rheumatology and chronic painful conditions, make up a significant proportion of this workload. The main musculoskeletal areas which GPs are referring to secondary care include hip and knee conditions, soinal conditions, shoulder and hand pathology, including carpal tunnel syndrome and trigger finger. To help better manage this workload within primary care we propose developing a new community-based monthly musculoskeletal clinic based within local GP surgeries, led by local GPs, supported by a MSK educational programme open to all GPs in the area. Outcomes which we will be monitored from this quality improvement work will include secondary care referrals and maintaining high patient satisfaction as well as improving GP confidence in managing MSK conditions.

\section{Acknowledgment}

The authors wish to acknowledge the Belfast GP Federation for their help throughout this project. This project could not have been completed without the support of all staff at the 2 GP surgeries as well as at orthopaedic ICATs, Southern Trust and therefore a big thank you must go to them.

\section{Funding}

This work was not supported by any external funding.

\section{References}

1. Jordan K, Kadam U, Hayward R, Porcheret M, Young C, et al. (2010) Annual consultation prevalence of regional musculoskeletal problems in primary care: an observational study. BMC musculoskeletal disorders 11: 144.

2. https://www.health-ni.gov.uk/

3. BMA (2015) General Practice in Crisis-A Report on Primary Care in Northern Ireland. British Medical Association.

4. Royal College of General Practitioners (2015) Delivering Change for General Practice: A Strategy for Improving Patient Care in Northern Ireland. RCGP NI. 
Citation: Heron N, Ryans I (2016) What Musculoskeletal (MSK) Conditions are Referred from Routine General Practice (GP) and what Impact does this have on Developing Innovative Care Models for Patients with MSK Conditions in Primary Care?. Int J Phys Med Rehabil 4: 375. doi:10.4172/2329-9096.1000375

Page 10 of 10

5. BMA (2015) General Practice in Northern Ireland: The Case for Change. British Medical Association.

6. Goff I, Wise E, Coady D, Walker D (2014) Musculoskeletal training: are GP trainees exposed to the right case mix for independent practice? Clin Rheumatol 35: 507-511.

7. Heron N (2015) Musculoskeletal (MSK) and Sport and Exercise Medicine (SEM) in General Practice (GP): A Novel GP-based MSK and SEM Clinic for Managing Musculoskeletal symptoms in a GP. BMJ Qual Improv Report 4.

8. Heron N (2015) A 'novel' model for integrating Sport and Exercise Medicine (SEM) and Musculoskeletal(MSK) management into primary care in the UK. BMJ Open Sport Exerc Med 1: e000027. 